\title{
Galilean Equivalence for Galactic Dark Matter
}

\author{
Michael Kesden ${ }^{1}$ and Marc Kamionkowski ${ }^{2}$ \\ ${ }^{1}$ Canadian Institute for Theoretical Astrophysics, University of Toronto, Toronto, ON M5S 3H8, Canada \\ ${ }^{2}$ California Institute of Technology, Mail Code 130-33, Pasadena, California 91125, USA \\ (Received 22 June 2006; revised manuscript received 2 August 2006; published 28 September 2006)
}

Satellite galaxies are tidally disrupted as they orbit the Milky Way. If dark matter (DM) experiences a stronger self-attraction than baryons, stars will preferentially gain rather than lose energy during tidal disruption, leading to an enhancement in the trailing compared to the leading tidal stream. The Sgr dwarf galaxy is seen to have roughly equal streams, challenging models in which DM and baryons accelerate differently by more than $10 \%$. Future observations and a better understanding of DM distribution should allow detection of equivalence violation at the percent level.

DOI: 10.1103/PhysRevLett.97.131303

Galileo showed at the Leaning Tower that objects of different masses and materials fall the same way in a gravitational field. This equivalence principle, later a cornerstone of Einstein's general relativity, has been tested repeatedly through a variety of experiments since Galileo's time and since the time of Einstein (see, e.g., Ref. [1]). These tests have confirmed that the materials found on Earth and in the Solar System all satisfy the equivalence principle to a remarkable degree. But what about the dark matter that fills galactic halos and dominates the mass density of the Universe? Is the dark matter in the Milky Way's halo accelerated the same as baryons in a gravitational field?

The simplest and most favored candidates for dark matter (DM), like weakly interacting massive particles (WIMPs) [2-4] and axions [5-7], do satisfy the equivalence principle (EP). However, there are a number of reasons to test this assumption. First of all, we still have no empirical evidence for the existence of WIMPs or axions. Some have argued that a stronger self-gravity for dark matter is required to clear dwarf galaxies from voids in the galaxy distribution [8-10]. Moreover, the recent discovery that the cosmological expansion is accelerating $[11,12]$ suggests that there may be more to gravity than general relativity - in particular, the quintessence field may mediate an additional long-range self-interaction between dark-matter particles $[13,14]$. There is thus considerable motivation to scrutinize our cherished notions about the equivalence principle.

Violations of the equivalence principle in the dark sector may be modeled phenomenologically by attributing to dark-matter particles $\psi$ a "fifth force" $[15,16]$,

$$
V_{\phi}(r)=-\frac{g^{2}}{4 \pi r} e^{-m_{\phi} r}
$$

Here $g$ is a dimensionless coupling constant, and $m_{\phi}$ is the mass of the scalar particle $\phi$ mediating the interaction. On scales $r \ll m_{\phi}^{-1}$, the potential of Eq. (1) leads to an
PACS numbers: 95.35.+d, 98.56.Wm, 98.62.Gq, 98.65.Fz

inverse-square-law force between DM particles of mass $m_{\psi}$ with a strength suppressed by a factor $\beta^{2}$ compared to gravity, where $\beta \equiv g m_{\mathrm{Pl}} / \sqrt{4 \pi} m_{\psi}$.

Several cosmological consequences of such a DM force have already been explored. First of all, to clear dwarf galaxies from voids, values $\beta \gtrsim 1$ and $m_{\phi}^{-1} \gtrsim 1 \mathrm{Mpc}$ are required [17]. An attractive force for $r \ll m_{\phi}^{-1}$ would enhance structure formation on these scales, leading to a corresponding increase in the density-perturbation power spectrum [18], an effect, though, that can be mimicked by a blue tilt in the power spectrum. An EP-violating coupling between DM and quintessence could also induce a scaleindependent bias between baryons and DM, though this effect is model dependent [14]. Refs. [15,18] had noted that a DM force would strip a baryonic core from its dark halo and applied this to typical galaxies in the Coma cluster to set a limit $\beta<2.2$. Clusters might also test an attractive DM force, as baryons would be preferentially lost compared to the more tightly bound DM during the mergers leading to their formation [19]. This test is complicated, however, by gas physics which is expected to reduce the cluster baryon-to-DM mass ratio below the cosmological value, even in the absence of a DM force.

In this Letter, we consider the effects of a DM force on galactic scales. We propose here that tidal streams produced by the disruption of a DM-dominated satellite galaxy orbiting in the halo of a much larger host galaxy provide a powerful probe of an EP-violating DM force. The reasoning follows by comparing the satellite's orbital energy $E_{\text {orb }}$, the energy $E_{\text {tid }}$ imparted during tidal disruption, and the self-binding energy $E_{\text {bin }}$ of the satellite [20],

$$
\begin{gathered}
E_{\mathrm{orb}}=\frac{G M_{R}}{R}, \\
E_{\mathrm{tid}}=r_{\mathrm{tid}} \frac{d \Phi_{\mathrm{host}}}{d R}=\left(\frac{m_{\mathrm{sat}}}{M_{R}}\right)^{1 / 3} E_{\mathrm{orb}},
\end{gathered}
$$




$$
E_{\text {bin }}=\frac{G m_{\text {sat }}}{r_{\text {sat }}}=\left(\frac{m_{\text {sat }}}{M_{R}}\right)^{2 / 3} E_{\text {orb }} .
$$

Here the host galaxy has a potential $\Phi_{\text {host }}(R)$ and mass $M_{R}$ within the satellite's orbit of radius $R$, and the satellite has a mass $m_{\text {sat }}$ and radius $r_{\text {sat }}$ which fill its tidal radius $r_{\text {tid }}$. When the satellite is much less massive than the host galaxy, $m_{\text {sat }} / M_{R} \ll 1$, a distinct hierarchy,

$$
E_{\text {orb }} \gg E_{\text {tid }} \gg E_{\text {bin }},
$$

exists in these three energy scales, implying that the disrupted stars and satellite will trace similar orbits in the host galaxy's potential regardless of the details of tidal disruption or the satellite's internal structure. The disrupted stars will act like purely baryonic test particles, while the satellite itself behaves largely like a DM test particle, if it is DM dominated.

Fortunately, the Sagittarius (Sgr) dwarf galaxy, the Milky Way's closest satellite at a Galactocentric distance of only $16 \mathrm{kpc}$, is nearly ideal for our purposes. The Sgr dwarf has extended leading and trailing tidal streams observed by the Two-Micron All Sky Survey (2MASS) [21] and the Sloan Digital Sky Survey (SDSS) [22]. Using a sample of over $1000 \mathrm{M}$-giant stars with a known colormagnitude relation, the 2MASS collaboration has measured not just surface brightnesses along the streams, but also distances and spectroscopic velocities as well [23]. Comparing these observations to simulations has led to estimates of the mass of the Sgr dwarf of $M_{\mathrm{Sgr}}=$ $(2-5) \times 10^{8} M_{\odot}$, mass-to-light ratio $M_{\mathrm{Sgr}} / L_{\mathrm{Sgr}}=$ $14-36 M_{\odot} / L_{\odot}$, and Sgr orbit with pericenter $10-19 \mathrm{kpc}$, apocenter $56-59 \mathrm{kpc}$, and period 0.85-0.87 Gyr [24]. The large mass-to-light ratio suggests that the Sgr dwarf is indeed DM dominated and therefore a suitable place to search for DM forces.

To study more carefully the effects of EP violation on tidal disruption, we performed our own simulations of the tidal disruption of a satellite with a mass $\left(5 \times 10^{8} M_{\odot}\right)$, mass-to-light ratio $\left(40 M_{\odot} / L_{\odot}\right)$, and orbit (pericenter $14 \mathrm{kpc}$, apocenter $59 \mathrm{kpc}$ ) similar to that of the Sgr dwarf. We could not compare our simulations directly with those of Ref. [24], as we performed $N$-body simulations of a Navarro-Frenk-White (NFW) profile for our Milky Way halos, and they used a static logarithmic potential. An active halo allows for dynamical friction over the course of the simulation and possible backreaction on the halo due to the DM force. While we did not attempt to reproduce the detailed features of the Sgr tidal streams, our simulations are sufficient to demonstrate that even a small DM force could have significant observational consequences. The initial conditions for our simulations were produced using GALACTICS [25], which makes use of phase-space distribution functions (DFs) that are analytic in the orbital energy and angular momentum. By Jeans' theorem, these DFs are equilibrium solutions to the collisionless Boltzmann equations [26], and they can be combined to produce realistic and stable models of the composite Milky Way bulge-disk-halo system [25]. We used the two Milky Way models of Ref. [25] that best fit observational constraints, including the Galactic rotation curve and local velocity ellipsoid. The simulations were evolved using a modified version of the $N$-body code GADGET-2 [27]. A more detailed description of our simulations are provided in Ref. [28].

Four simulations of tidal disruption are depicted in Fig. 1, with DM forces given by Eq. (1) with different values of the charge-to-mass ratio $\beta$. The scalar field is assumed massless $\left(m_{\phi}=0\right)$, so the DM force is a true inverse square law. The ratio $\beta$ increases from 0.0 at bottom left to 0.3 at top left as one proceeds counterclockwise. The simulations begin with the satellite at apocenter $59 \mathrm{kpc}$ from the Galactic center and last for $2.4 \mathrm{Gyr}$ (almost three full orbits). The tangential velocities are adjusted so that all orbits are projected to have a pericenter of $14 \mathrm{kpc}$. The orbits are counterclockwise in the $x-z$ plane so that the edge of the leading stream appears at 12 o'clock with respect to the Galactic center in Fig. 1, while the edge of the trailing stream is at about 10 o'clock. The Sgr dwarf is modeled with a truncated NFW profile for both stars and

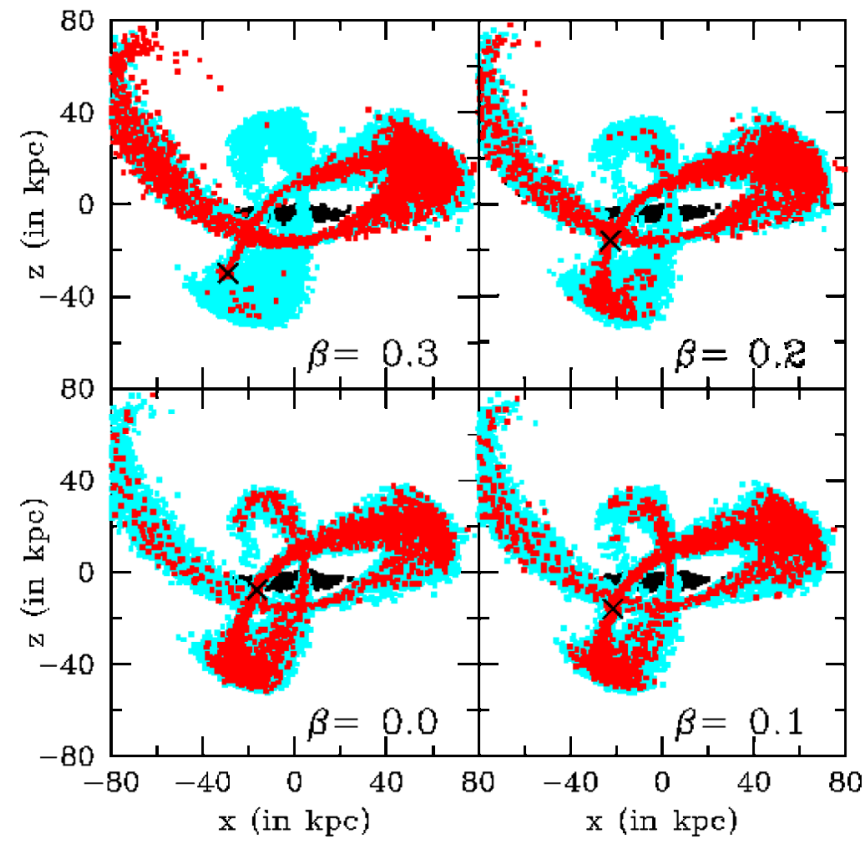

FIG. 1 (color online). Simulations of the tidal disruption of a satellite galaxy in the presence of a dark matter force. The charge-to-mass ratio $\beta$ increases from 0.0 in increments of 0.1 , going counterclockwise from the bottom left. The Galactic disk is in black. Sgr stars are shown in red (dark gray) while the Sgr dark matter is blue (light gray). The tidal streams are projected onto the orbital plane. Orbits are counterclockwise; the upper left figure shows that for $\beta=0.3$ (a dark-matter force 9\% the strength of gravity) stars are almost absent from the leading stream (at 12 o'clock with respect to the Galactic center). $X$ 's denote the location of the bound Sgr core. 
DM, in keeping with the simulations of Ref. [24], where it was concluded that observations could not yet determine distinct profiles for the two components. Thus, the stars shown in red (dark gray) in the bottom left panel are simply a downsampling of the DM distribution illustrated in blue (light gray).

As the DM force increases in strength, the leading stream is systematically depleted of stars, while the trailing stream is correspondingly enhanced. By the time $\beta$ reaches 0.3 in the top left panel, the leading stream is virtually devoid of stars. The primary reason for this effect is that in the presence of an attractive DM force, the center of mass of the satellite's stars is displaced outwards with respect to that of its DM. The bound stars lie at the bottom of the satellite's gravitational potential well and are therefore forced to orbit the Galactic center at the same speed as the DM. However, they do not have the attractive pull of the DM force from the Milky Way's halo to supplement gravity in providing the required centripetal force. The stars are therefore displaced outwards so that the inward gravitational pull of the satellite's DM can provide this additional centripetal force. From this outer position, stars are more likely to be tidally disrupted from the far side of the satellite than the side closest to the Galactic center. Stars disrupted from the far side gain energy and are boosted onto higher orbits in the Milky Way's potential well where their angular velocity is slower than that of the satellite. They therefore trail behind the satellite and develop into a trailing tidal stream. A repulsive DM force will induce an opposite effect, displacing the stars towards the Galactic center and preferentially creating a leading rather than a trailing tidal stream.

This asymmetry in the leading compared to the trailing tidal streams is a distinctive signature of a DM force that can be observed in the stellar densities measured along the stream. The normalized stellar densities of the four simulations presented in Fig. 1 are shown in the four curves of Fig. 2. Orbits in our composite Milky Way model do not close, and the four peaks in the stellar density distribution correspond to the four apocenter passages appearing at 2, 7, 10, and 12 o'clock in Fig. 1. Orbital velocities are minimized at apocenter, so stars tend to accumulate there. The ratio of the number of stars near the apocenters furthest along the leading and trailing tidal streams thus provides a convenient measure of the asymmetry between the streams. The ratio of the number of stars in the leading segment stretching from $-300^{\circ}$ to $-200^{\circ}$ as compared to the trailing segment from $350^{\circ}$ to $450^{\circ}$ drops from 0.66 in the absence of a DM force down to 0.44, 0.091, and 0.0042 for $\beta=0.1,0.2$, and 0.3 as indicated by the solid black curve in Fig. 3. The SDSS has observed hundreds of stars per square degree along the Sgr tidal stream [22]. As the Sgr dwarf is observed to have an extensive leading stream, we conclude that a DM force as weak as $9 \%$ the strength of gravity is likely to be observationally unacceptable, in

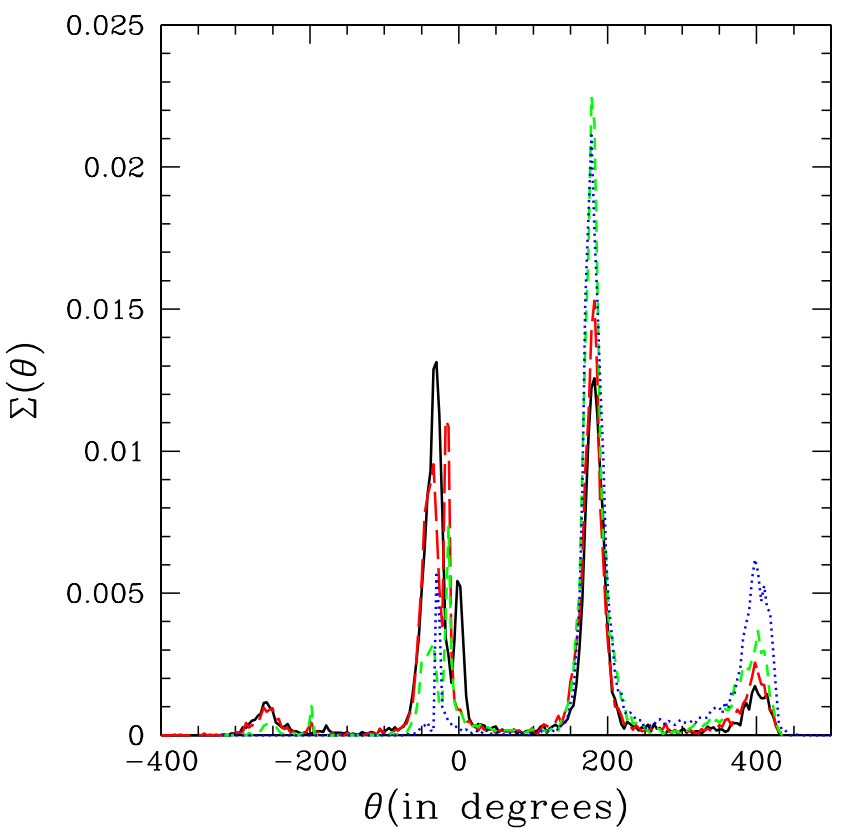

FIG. 2 (color online). Surface density of stars as a function of angular distance $\theta$ along the tidal stream. The satellite core is located at $0^{\circ}$, while the trailing and leading streams are at positive and negative $\theta$, respectively. As the tidal streams wrap around the Galaxy more than once, $\theta$ extends beyond $\pm 180^{\circ}$. The four curves correspond to the four panels of Fig. 1, with black (solid), red (long-dashed), green (short-dashed), and blue (dotted) curves belonging to the $\beta=0.0$ through 0.3 simulations.

which case the proposal $[9,10,17]$ that EP-violating dark matter clears dwarf galaxies from voids would be ruled out.

Our simulations suggest that current observations can already place impressive constraints on a DM force, but several concerns remain before we can confront our simulations with data. If the stream wraps around the Galaxy more than once, we must be able to distinguish true leading stars from trailing stars that have almost been lapped by the satellite. We have been able to do this surprisingly well in simulations using only the radial velocities, distances, and positions along the stream. As 2MASS has collected this data, identifying leading and trailing stars should already be feasible and can certainly be accomplished by a future high-precision astrometry experiment like the Space Interferometry Mission (SIM) or Gaia. More troublesome is whether some other change in our Sgr or Milky Way models could produce the same asymmetric tidal tails that we are claiming as a signature of a DM force. Future investigation of this concern is certainly needed, and we have made a first attempt at this in Ref. [28], the results of which are summarized in Fig. 3. This signature is seen to be robust to changes in the Milky Way model and the mass, orbit, and phase-space distribution of the Sgr dwarf. In the absence of a DM force, the leading-to-trailing ratio exceeds 0.5 for all our models, while for $\beta>0.2$, the ratio is always below 0.2 . The detailed morphology of the stream 


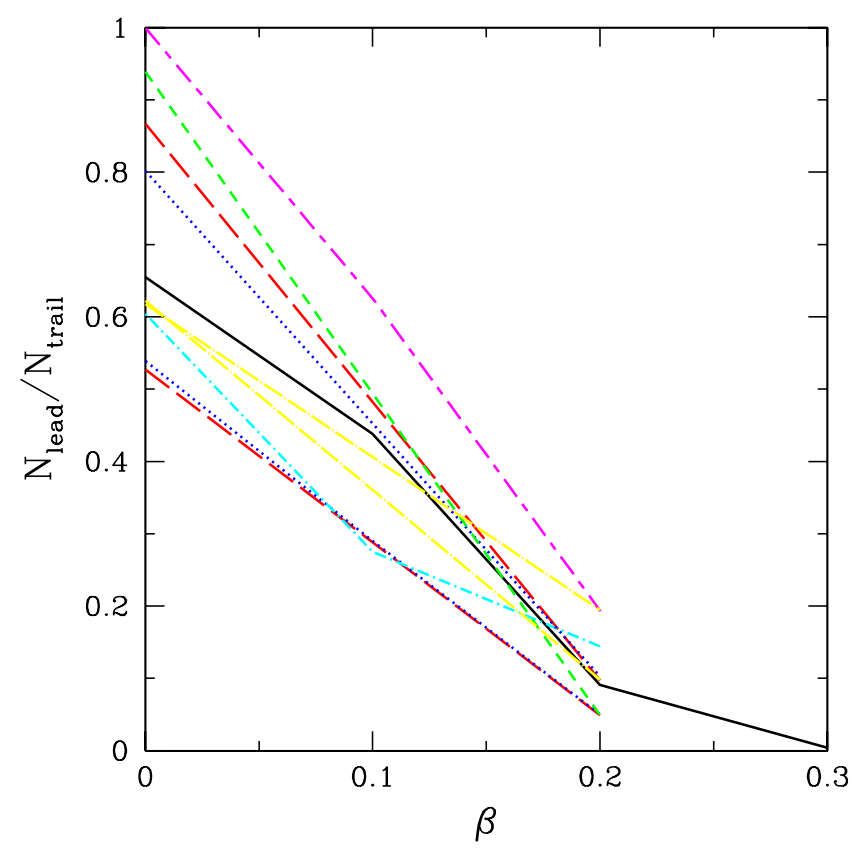

FIG. 3 (color online). The ratio of leading to trailing stars as a function of charge-to-mass ratio $\beta$ for different models of the host-satellite system. The black (solid) curve is our default bestfit model. The magenta (long-short-dashed) curve doubles the initial mass of the satellite. The two red (long-dashed) curves have rotating satellites: the top is prograde and the bottom is retrograde. The blue (dotted) curves have satellites with different orbits: the top curve has a more circular orbit, while the bottom curve has a planar orbit rather than the polar orbit of Sgr. The cyan (dot-short-dashed) curve uses a Milky Way model with lighter halo and heavier disk. The green (short-dashed) curve has a satellite where $25 \%$ of the most bound particles represent stars. The yellow (dot-long-dashed) curves have satellites with lower $M / L$ ratios (higher stellar mass fractions): the top curve has $M / L=4.5$ while the bottom curve has $M / L=10$.

also allows us to anticipate when the ratio should be high or low, making our test more sensitive than a single number. Our simulations suggest that it may be possible to detect a DM force a few percent the strength of gravity. We may not be able to drop DM off the Leaning Tower of Pisa, but the Sgr tidal streams may be the next best thing.

We wish to thank John Dubinski and Larry Widrow for assistance with the use of GALACTICS and Pat McDonanld, Neal Dalal, Christoph Pfrommer, and Jonathan Sievers for useful conversations. All computations were performed on CITA's McKenzie cluster [29], which was funded by the Canada Foundation for Innovation and the Ontario Innovation Trust. Kesden acknowledges support from the NASA Graduate Research Program, and NSERC of Canada. Kamionkowski acknowledges sup- port from the DoE No. DE-FG03-92-ER40701, NASA No. NNG05GF69G, and the Gordon and Betty Moore Foundation.

[1] C. M. Will, Living Rev. Relativity 4, 4 (2001).

[2] G. Jungman, M. Kamionkowski, and K. Griest, Phys. Rep. 267, 195 (1996).

[3] L. Bergstrom, Rep. Prog. Phys. 63, 793 (2000).

[4] G. Bertone, D. Hooper, and J. Silk, Phys. Rep. 405, 279 (2005).

[5] M. S. Turner, Phys. Rep. 197, 67 (1990).

[6] G. G. Raelt, Phys. Rep. 198, 1 (1990).

[7] L. J. Rosenberg and K. A. van Bibber, Phys. Rep. 325, 1 (2000).

[8] P. J. E. Peebles, Astrophys. J. 557, 495 (2001).

[9] S. S. Gubser and P. J. E. Peebles, Phys. Rev. D 70, 123511 (2004).

[10] S. S. Gubser and P. J. E. Peebles, Phys. Rev. D 70, 123510 (2004).

[11] S. Perlmutter et al. (Supernova Cosmology Project), Astrophys. J. 517, 565 (1999).

[12] A. G. Riess et al. (Supernova Search Team), Astron. J. 116, 1009 (1998).

[13] G. R. Farrar and P. J.E. Peebles, Astrophys. J. 604, 1 (2004).

[14] L. Amendola and D. Tocchini-Valentini, Phys. Rev. D 66, 043528 (2002).

[15] J. A. Frieman and B. A. Gradwohl, Phys. Rev. Lett. 67, 2926 (1991).

[16] J. A. Frieman and B. A. Gradwohl, Science 260, 1441 (1993).

[17] A. Nusser, S. S. Gubser, and P. J. E. Peebles, Phys. Rev. D 71, 083505 (2005).

[18] B.-A. Gradwohl and J. A. Frieman, Astrophys. J. 398, 407 (1992).

[19] R. Mainini and S. Bonometto, Phys. Rev. D 74, 043504 (2006).

[20] K. V. Johnston, H. Zhao, D. N. Spergel, and L. Hernquist, Astrophys. J. 512, L109 (1999).

[21] S. R. Majewski, M. F. Skrutskie, M. D. Weinberg, and J. C. Ostheimer, Astrophys. J. 599, 1082 (2003).

[22] V. Belokurov et al., Astrophys. J. 642, L137 (2006).

[23] S. R. Majewski et al., Astron. J. 128, 245 (2004).

[24] D. R. Law, K. V. Johnston, and S. R. Majewski, Astrophys. J. 619, 807 (2005).

[25] L. M. Widrow and J. Dubinski, Astrophys. J. 631, 838 (2005).

[26] K. Kuijken and J. Dubinski, Mon. Not. R. Astron. Soc. 277, 1341 (1995).

[27] V. Springel, Mon. Not. R. Astron. Soc. 364, 1105 (2005).

[28] M. Kesden and M. Kamionkowski (to be published).

[29] J. Dubinski, R. Humble, U.-L. Pen, C. Loken, and P. Martin, astro-ph/0305109. 